\title{
Analysis of the Related Risk Factors of Inguinal Lymph Node Metastasis in Patients with Penile Cancer: A Cross-sectional Study
}

yatao jia ( $D 2549118761 @ q q . c o m)$

Jilin University First Hospital

Hongwei Zhao

the first hospital of jilin university

\section{Yun Hao}

Jilin University First Hospital

Jiang Zhu

Baoji People's Hospital

Yingyi Li

Baoji People's Hospital

\section{Yanbo Wang}

Jilin University First Hospital

\section{Research article}

Keywords: Penile cancer, Inguinal lymph nodes metastasis, predictor, Inguinal lymph nodes dissection

Posted Date: May 18th, 2021

DOl: https://doi.org/10.21203/rs.3.rs-531246/v1

License: (1) This work is licensed under a Creative Commons Attribution 4.0 International License.

Read Full License

Version of Record: A version of this preprint was published at International braz j urol on April 1st, 2022.

See the published version at https://doi.org/10.1590/s1677-5538.ibju.2021.0613. 


\section{Abstract}

Background: To determine independent predictors of inguinal lymph node(ILN) metastasis in patients with penile-cancer.

Patients and methods: We retrospectively analyzed all patients with penile-cancer undergoing surgery at our medical center in ten years $(\mathrm{N}=157)$. Using univariate and multivariate logistic-regression models, we assessed associations between the following factors: age, medical-history, phimosis, onset-time, number and maximum diameter of involved ILNs, pathological T stage, degree of tumor differentiation and/or cornification, lymphatic vascular infiltration(LVI), nerve infiltration, and ILN metastases. Interaction and stratified analyses were then used to assess age, phimosis, onset-time, number of ILNs, cornification, and nerve infiltration.

Results: Ultimately, 110 patients were included. Multiple logistic-regression analysis showed that the following factors were significantly correlated with ILN metastasis: maximum diameter of enlarged ILNs, T stage, pathological differentiation, and LVI. Among patients with a maximum ILN diameter of $\geq 1.5 \mathrm{~cm}$, $50 \%(19 / 38)$ had LNM(HR=2.3, 95\% Cl: $1.0-5.1)$, whereas only $30.6 \%(22 / 72)$ of patients with a maximum ILN diameter $<1.5 \mathrm{~cm}$ showed LNM. Among 44 patients with stage Ta/T1, 10(22.7\%) showed ILN metastases, while 31 of $66(47.0 \%)$ patients with stage T2 showed ILN metastases(HR=3.0, 95\% Cl: $1.3-$ 7.1). Among 40 patients with highly differentiated penile-cancer, eight(20\%) showed ILN metastasis, while 33 of $70(47.1 \%)$ patients with low-to-middle differentiation showed ILN metastases(HR=3.6, 95\%Cl: 1.48.8). In the LVI-free group, the rate of LNM was $33.3 \%(32 / 96)$, whereas it was $64.3 \%(9 / 14)$ in the LVI group(HR=3.6, 95\% Cl: 1.1-11.6).

Conclusion: Our single-center results suggested that maximum ILN diameter, pathological T stage, pathological differentiation, and LVI were independent risk factors for ILN metastases.

\section{Introduction}

Penile cancer is a rare malignancy, with an incidence of 0.081 per 100,000 in the United States and Europe [1, 2], and a prevalence of 2.3 to 8.3 per 100,000 in some developing regions, such as Asia and parts of Africa [3]. Penile cancer has high malignancy and mainly spreads by lymphatic metastasis. The first station is the inguinal lymph nodes (ILNs), and jump metastasis rarely occurs [4]. ILN metastasis is the most important determinant of treatment and prognosis in patients with penile cancer [3].

Pathology after lymph node biopsy or lymph node dissection is still the gold standard to evaluate ILN metastasis. However, it is an invasive operation that involves many postoperative complications, including poor lymph node drainage and poor wound healing [5]. Therefore, researchers must explore the risk factors for ILN metastasis to determine which patients with penile cancer require ILN dissection. In so doing, patients with occult metastasis could receive prompt treatment, while patients with a lower risk of ILN metastasis could avoid excessive treatment. 
Tumor stage, histological grade, lymphatic and vascular infiltration, histological subtype, human papillomavirus, etc. have been identified as important predictors of ILN metastasis in previous studies [6, 7]. However, these studies were carried out in a single center, and the sample size was small. In addition, only univariate analysis was used to explore the risk factors for ILN metastasis. In the present study, to better explore the risk factors for ILN metastasis, we conducted multiple logistic analysis, subgroup analysis, and interaction tests on all patients with penile cancer undergoing surgery in a large tertiary hospital over a 10-year period.

\section{Patients And Methods}

This cross-sectional study was conducted between January 2010 and December 2019 in a comprehensive tertiary hospital in China. The inclusion criteria were as follows: (1) primary tumor treated surgically; (2) tumor pathology confirmed by experienced pathologists; (3) ILN metastasis pathologically confirmed by biopsy or prophylactic inguinal lymphadenectomy, or found based on clinical features during follow-up. Patients with pelvic lymph node or distant metastases were excluded, as were those treated in other hospitals.

We retrieved the following clinical information from the patients' medical records, including age, previous medical history (hypertension, diabetes, or cardiovascular disease), phimosis, onset time, number and maximum diameter of the involved ILNs, pathological T stage, degree of tumor differentiation and/or cornification, lymphatic vascular infiltration (LVI), and nerve infiltration. The number and maximum diameter of the ILNs were determined using ultrasound or computed tomography. Tumor stage was assessed according to the TNM system, which was updated in 2018 [8]. The pathological differentiation of tumors was evaluated according to the criteria of Velazquez et al. [9].

Primary tumors were treated surgically using either penis-sparing, partial amputation, or total excision via perineal urethrostomy. According to the EAU guidelines (2009) [10], ILN biopsy or bilateral ILN dissection are recommended for patients with stage $\geq T 1 G 2$ and/or enlarged lymph nodes that have not significantly shrunk after 4-6 weeks of antibiotic treatment. Surgery was performed by three experienced urologists. Informed consent was obtained from all patients, and the protocol was approved by the Institutional Ethics Committee of The First Hospital of Jilin University, Jilin, China.

Continuous variables are presented as mean \pm standard deviation (SD) or median (interquartile range), while categorical variables are written as frequency or percentage. To facilitate statistical analysis, all continuous variables except age were converted into categorical variables. Related risk factors for penile cancer were identified using univariate logistic regression analyses, and independent predictive factors of ILN metastasis were confirmed by multivariate logistic regression analyses. We used the Statistical Packages R (The R Foundation, Vienna, Austria) to analyze the data. When the P-value was less than 0.05 , the statistical difference was considered significant.

\section{Results}


A total of 157 patients were identified; 47 were excluded due to lack of follow-up data, resulting in 110 patients included in the study (Fig. 1). Forty-one patients had confirmed ILN metastasis, of whom 25 were pathologically confirmed by ILN biopsy, eight were confirmed by pathology after prophylactic inguinal lymphadenectomy, and eight were found during follow-up. There were no signs of ILN metastasis in 69 cases. Hence, the ILN metastasis rate was $37.3 \%$.

Table 1 lists the clinicopathological characteristics and univariate analysis of variables associated with ILN metastasis in the 110 patients. The mean age was $61.6 \pm 11.8$ years. Univariate analysis showed that the following factors were correlated with ILN metastasis: maximum diameter of enlarged ILNs (P = 0.045), pathological stage $(P=0.010)$, degree of pathological differentiation $(P=0.009)$, and LVI $(P=$ $0.025)$. 
Table 1

Clinicopathological characteristics and univariate analysis of variables associated with inguinal lymph node metastasis

\begin{tabular}{|c|c|c|c|c|c|}
\hline & Total & $\begin{array}{l}\text { Without } \\
\text { metastasis }\end{array}$ & $\begin{array}{l}\text { With } \\
\text { metastasis }\end{array}$ & $\mathrm{HR}(95 \% \mathrm{Cl})$ & $\begin{array}{l}\mathrm{P}- \\
\text { value }\end{array}$ \\
\hline$N$ & 110 & 69 & 41 & & \\
\hline $\begin{array}{l}\text { Age(years)(Mean + } \\
\text { SD) }\end{array}$ & $\begin{array}{l}61.6 \pm \\
11.8\end{array}$ & $61.0 \pm 12.0$ & $62.5 \pm 11.5$ & $\begin{array}{l}0.1(-0.3, \\
0.5)\end{array}$ & 0.529 \\
\hline $\operatorname{HBP}(\mathrm{N}, \%)$ & & & & $\begin{array}{l}0.0(-0.4 \\
0.4)\end{array}$ & 0.984 \\
\hline no & 94 (85.5\%) & 59 (85.5\%) & 35 (85.4\%) & & \\
\hline yes & $16(14.5 \%)$ & $10(14.5 \%)$ & $6(14.6 \%)$ & & \\
\hline Diabetes(N,\%) & & & & $\begin{array}{l}0.1(-0.3 \\
0.4)\end{array}$ & 0.799 \\
\hline no & $\begin{array}{l}101 \\
(91.8 \%)\end{array}$ & $63(91.3 \%)$ & 38 (92.7\%) & & \\
\hline yes & $9(8.2 \%)$ & $6(8.7 \%)$ & $3(7.3 \%)$ & & \\
\hline $\operatorname{CDV}(\mathrm{N}, \%)$ & & & & $\begin{array}{l}0.1(-0.2 \\
0.5)\end{array}$ & 0.439 \\
\hline no & $\begin{array}{l}102 \\
(92.7 \%)\end{array}$ & 65 (94.2\%) & 37 (90.2\%) & & \\
\hline yes & $8(7.3 \%)$ & $4(5.8 \%)$ & $4(9.8 \%)$ & & \\
\hline Phimosis $(\mathrm{N}, \%)$ & & & & $\begin{array}{l}0.2(-0.2 \\
0.6)\end{array}$ & 0.347 \\
\hline no & 58 (52.7\%) & $34(49.3 \%)$ & $24(58.5 \%)$ & & \\
\hline yes & $52(47.3 \%)$ & 35 (50.7\%) & $17(41.5 \%)$ & & \\
\hline $\begin{array}{l}\text { Onset_time(month) } \\
(\mathrm{N}, \%)\end{array}$ & & & & $\begin{array}{l}0.1(-0.2 \\
0.5)\end{array}$ & 0.475 \\
\hline$<12$ & 76 (69.1\%) & $46(66.7 \%)$ & 30 (73.2\%) & & \\
\hline$>=12$ & $34(30.9 \%)$ & $23(33.3 \%)$ & $11(26.8 \%)$ & & \\
\hline
\end{tabular}

The $P$ value is written in italics when it is less than 0.05

HBP: high blood pressure; CDV: cardiovascular disease;ILN: Inguinal lymph node;LVI: Lymphatic vascular infiltration, T stage: The TNM system of penile cancer updated in 2018; degree of tumor differentiation: According to the percentage of undifferentiated cells, the tumor was divided into middle and low differentiated groups and highly differentiated groups. Cl: confidence interval; HR: hazard ratio; 


\begin{tabular}{|c|c|c|c|c|c|}
\hline & Total & $\begin{array}{l}\text { Without } \\
\text { metastasis }\end{array}$ & $\begin{array}{l}\text { With } \\
\text { metastasis }\end{array}$ & $\mathrm{HR}(95 \% \mathrm{Cl})$ & $\begin{array}{l}\mathrm{P}- \\
\text { value }\end{array}$ \\
\hline Number_ILN(N,\%) & & & & $\begin{array}{l}0.2(-0.2, \\
0.6)\end{array}$ & 0.254 \\
\hline$\otimes 3$ & $46(41.8 \%)$ & $26(37.7 \%)$ & $20(48.8 \%)$ & & \\
\hline$\geq 3$ & $64(58.2 \%)$ & $43(62.3 \%)$ & $21(51.2 \%)$ & & \\
\hline Maxium_ILN(N,\%) & & & & $\begin{array}{l}0.4(0.0 \\
0.8)\end{array}$ & 0.045 \\
\hline$₫ 1.5 \mathrm{~cm}$ & $72(65.5 \%)$ & $50(72.5 \%)$ & $22(53.7 \%)$ & & \\
\hline$\geq 1.5 \mathrm{~cm}$ & $38(34.5 \%)$ & $19(27.5 \%)$ & $19(46.3 \%)$ & & \\
\hline T_stage $(\mathrm{N}, \%)$ & & & & $\begin{array}{l}0.5(0.1 \\
0.9)\end{array}$ & 0.01 \\
\hline $\mathrm{Ta} / \mathrm{T} 1$ & $44(40.0 \%)$ & $34(49.3 \%)$ & $10(24.4 \%)$ & & \\
\hline T2 and higher & $66(60.0 \%)$ & $35(50.7 \%)$ & $31(75.6 \%)$ & & \\
\hline Differentiation(N,\%) & & & & $\begin{array}{l}0.6(0.2 \\
1.0)\end{array}$ & 0.005 \\
\hline Lower-middle & $70(63.6 \%)$ & $37(53.6 \%)$ & $33(80.5 \%)$ & & \\
\hline higher & $40(36.4 \%)$ & $32(46.4 \%)$ & $8(19.5 \%)$ & & \\
\hline Cornification(N,\%) & & & & $\begin{array}{l}0.0(-0.3 \\
0.4)\end{array}$ & 0.807 \\
\hline no & $74(67.3 \%)$ & $47(68.1 \%)$ & $27(65.9 \%)$ & & \\
\hline yes & $36(32.7 \%)$ & $22(31.9 \%)$ & $14(34.1 \%)$ & & \\
\hline $\operatorname{LVI}(\mathrm{N}, \%)$ & & & & $\begin{array}{l}0.4(0.0 \\
0.8)\end{array}$ & 0.025 \\
\hline no & $96(87.3 \%)$ & $64(92.8 \%)$ & $32(78.0 \%)$ & & \\
\hline yes & $14(12.7 \%)$ & $5(7.2 \%)$ & $9(22.0 \%)$ & & \\
\hline Nerve_infiltration(N,\%) & & & & $\begin{array}{l}0.2(-0.2 \\
0.6)\end{array}$ & 0.362 \\
\hline no & 92 (83.6\%) & $56(81.2 \%)$ & 36 (87.8\%) & & \\
\hline
\end{tabular}

The $P$ value is written in italics when it is less than 0.05

HBP: high blood pressure; CDV: cardiovascular disease;ILN: Inguinal lymph node;LVI: Lymphatic vascular infiltration, T stage: The TNM system of penile cancer updated in 2018; degree of tumor differentiation: According to the percentage of undifferentiated cells, the tumor was divided into middle and low differentiated groups and highly differentiated groups. Cl: confidence interval; HR: hazard ratio; 


\begin{tabular}{|llllll|}
\hline & Total & $\begin{array}{l}\text { Without } \\
\text { metastasis }\end{array}$ & $\begin{array}{l}\text { With } \\
\text { metastasis }\end{array}$ & HR(95\%Cl) & $\begin{array}{l}\text { P- } \\
\text { value }\end{array}$ \\
\hline yes & $18(16.4 \%)$ & $13(18.8 \%)$ & $5(12.2 \%)$ & \\
\hline
\end{tabular}

The $P$ value is written in italics when it is less than 0.05

HBP: high blood pressure; CDV: cardiovascular disease;ILN: Inguinal lymph node;LVI: Lymphatic vascular infiltration, T stage: The TNM system of penile cancer updated in 2018; degree of tumor differentiation: According to the percentage of undifferentiated cells, the tumor was divided into middle and low differentiated groups and highly differentiated groups. Cl: confidence interval; HR: hazard ratio;

Significant single factors were included in the multivariate analysis, as shown in Table 2. We applied both non-adjusted and multivariate adjusted models (adjusted for age, previous medical history, and other variables that affected the $X$ regression coefficient by more than $10 \%$ ). A two-sided significance level of 0.05 was used to evaluate statistical significance. The results showed that the following factors were independent predictors of ILN metastasis: largest diameter of enlarged ILNs, T stage of tumor, pathological differentiation, and LVI. Specifically, patients whose largest ILN diameter was $\geq 1.5 \mathrm{~cm}$ showed a 1.3-fold increased risk of metastasis over those whose largest ILN diameter was $<1.5 \mathrm{~cm}$. Those with tumor stage T2 and above showed two-fold greater risk of ILN metastasis than those with tumor stage Ta or T1. Those with low to moderate tumor differentiation had a 2.6-fold greater risk of ILN metastasis than those with high pathological differentiation. Finally, those with LVI had a 2.6-fold greater risk of ILN metastasis than those without. 
Table 2

Multiple logistic regression models assessed the correlation between risk factors and ILN metastasis

\begin{tabular}{|c|c|c|c|}
\hline Exposure & Non-adjusted & Adjust I & Adjust II \\
\hline & HR,95\%CI P value & $\mathrm{HR}, 95 \% \mathrm{Cl} \mathrm{P}$ value & $\mathrm{HR}, 95 \% \mathrm{Cl} P$ value \\
\hline \multicolumn{4}{|l|}{ Maxium_ILN } \\
\hline$\bigotimes 1.5 \mathrm{~cm}$ & 1 & 1 & 1 \\
\hline$\geq 1.5 \mathrm{~cm}$ & $2.3(1.0,5.1) 0.047$ & $2.4(1.1,5.6) 0.035$ & $10.7(2.1,53.3) 0.004$ \\
\hline \multicolumn{4}{|l|}{ T_stage } \\
\hline $\mathrm{Ta} / \mathrm{T} 1$ & 1 & 1 & 1 \\
\hline T2 and above & $3.0(1.3,7.1) 0.011$ & $3.1(1.3,7.5) 0.010$ & $7.1(1.7,28.9) 0.006$ \\
\hline \multicolumn{4}{|l|}{ Differentiation } \\
\hline high & 1 & 1 & 1 \\
\hline low-middle & $3.6(1.4,8.8) 0.006$ & $4.0(1.5,10.4) 0.004$ & $6.2(1.9,20.2) 0.003$ \\
\hline \multicolumn{4}{|l|}{ LVI } \\
\hline no & 1 & 1 & 1 \\
\hline yes & $3.6(1.1,11.6) 0.032$ & $3.6(1.1,11.8) 0.033$ & $7.4(1.3,40.8) 0.022$ \\
\hline \multicolumn{4}{|c|}{ 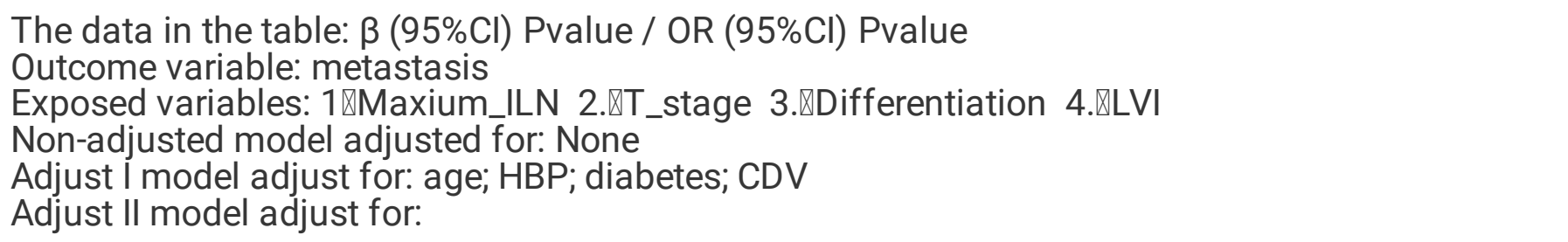 } \\
\hline \multicolumn{4}{|c|}{ 1囚age; HBP; diabetes; CDV;+onset_time; T_stage; differentiation; LVI; Number_ILN } \\
\hline \multicolumn{4}{|c|}{$\begin{array}{l}\text { 2) age; HBP; diabetes; CDV+onset_time; Number_ILN; Maxium_ILN; differentiation; LVI; nerve } \\
\text { infiltration }\end{array}$} \\
\hline \multicolumn{4}{|c|}{ 3\age; HBP; diabetes; CDV+onset_time; Number_ILN; Nerve_infiltration } \\
\hline \multicolumn{4}{|c|}{$\begin{array}{l}\text { 4囚age, HBP, diabetes, CDV+onset_time, Number_ILN, Maxium_ILN, T_stage, Nerve_infiltration, } \\
\text { differentiation }\end{array}$} \\
\hline ILN: inguinal ly & nodes; LVI: lymphatic & lar infiltration & \\
\hline
\end{tabular}

To further demonstrate the stability of our results, we performed stratified analyses and interaction tests of the four independent risk factors, as shown in the forest plot (Fig. 2:a-d) and additional file 1. The results showed no significant interactions.

\section{Discussion}


The most important factor affecting the prognosis of penile cancer is ILN metastasis [3, 11-13]. Several studies have indicated that the rate of ILN metastasis in patients with penile cancer is $30-60 \%$ [14]. The latest meta-analysis [15] selected 42 eligible studies that included a total of 4,802 patients, of whom $1,706(36 \%)$ were diagnosed with ILN metastasis. This finding was corroborated by our results, in which $37.3 \%$ of patients had ILN metastasis (41/110).

In our multiple regression analysis, the maximum diameter of the enlarged ILNs, pathological stage, pathological differentiation, and LVI were the only predictors of ILN metastasis. We conducted a stratified analysis and interaction tests on the four factors and did not find any obvious interaction, further proving the stability of our results.

A maximum ILN diameter of $>1.0 \mathrm{~cm}$ is usually considered abnormal, while a diameter $>1.5 \mathrm{~cm}$, with a relatively hard texture, strongly indicates tumor metastasis [16]. The present study suggested that the largest diameter of the enlarged ILNs was an independent risk factor, corroborating studies by Tang et al. [17] and Zhou et al. [6]. However, in another study, 50\% of enlarged ILNs were inflamed or reactive, rather than metastatic [14], indicating that ILN metastasis cannot be reliably detected using imaging or clinical evaluation. It is essential to predict ILNM in combination with the pathological characteristics of the primary tumor.

The pathological stage of the primary tumor is generally considered the most important parameter for predicting ILN metastasis in patients with penile cancer [14, 18]. Depending on whether there is infiltration of the urethra or corpus cavernosum, the tumor stage can be classified as T1, T2, or higher [8]. In the present study, the rate of ILN metastasis in patients with T1 stage was $22.7 \%$ (10/44), while it was $47.0 \%$ (31/66) in those with T2 stage and above. The ILN metastasis rate in patients with T2 stage and above was significantly higher than that of patients with T1 stage disease. Several researchers have opined that, if the corpus cavernosum is infiltrated, ILN dissection should be performed, even if there are no obvious enlarged ILNs $[19,20]$. However, deciding to perform ILN dissection on all patients with T2 and above only based on tumor stage, which will lead to overtreatment. In the present study, $53.0 \%$ of patients with T2 stage and above showed no obvious signs of ILN metastasis. Therefore, other factors should be taken into account when screening high-risk patients.

The degree of tumor differentiation under pathology is negatively correlated with tumor pathological grade or malignancy, with lower tumor differentiation indicating higher grade, higher malignancy, and greater risk of metastasis [15]. Solsona et al. [21] found that the grade of tumor differentiation correlates well with ILN metastasis, corroborating our multiple regression analysis ( $\mathrm{HR}=3.0,95 \% \mathrm{Cl}$ : 1.3-7.1). According to Horenblas et al. [22], the lymph node metastasis rates of G1, G2, and G3 were $29 \%, 46 \%$, and $82 \%$, respectively. Our results were similar to these, with $20 \%(8 / 40), 45 \%(30 / 66)$, and $75 \%(3 / 4)$, respectively. Theodoresu et al. [23] asserted that tumor grade is the only predictor of ILN metastasis, and they recommended ILN dissection in patients with G2 and G3, which coincides with our point of view.

The existing literature has different opinions on whether LVI is a predictor of ILN metastasis. Some studies have ranked LVI as one of the most important factors of metastasis [24-27], while others have 
not [28]. Our findings suggest that LVI is significantly associated with ILN metastasis $(\mathrm{HR}=3.6,95 \% \mathrm{Cl}$ : 1.1-11.6) among patients with penile cancer.

The present study had several strengths. Firstly, although some potential confounding factors were unavoidable, we used strict statistical adjustment to minimize residual confounding. Secondly, the effect modifier factor analysis took full advantage of the data; no interaction was found, indicating that the results are more stable.

Conversely, there were some limitations of our study. The patients with penile cancer were collected from a large single medical center. Therefore, external validation of the results is required. The study was cross-sectional, and no information was available about the degree of risk factors prior to ILN metastasis, because the earlier pathology reports contained no such data. Moreover, no data were available regarding lymph node extranodal transfer and the growth pattern of tumors in some patients (papillary, ulcerated, invasive, etc.) As such, we could not consider these variables in the final results, although there was evidence that these factors had prognostic significance. Despite these limitations, the findings of this study have implications for clinicians when formulating further treatment plans in patients with penile cancer who have undergone surgery. However, prospective studies with more samples are needed.

\section{Conclusion}

In conclusion, the maximum diameter of the enlarged ILN, pathological stage, pathological differentiation, and LVI are independent predictive factors that worsen prognosis in patients with penile cancer. Specifically, patients with enlarged lymph nodes $>1.5 \mathrm{~cm}$ in diameter, pathological stage T2 and above, low-to-middle differentiation, and LVI are more likely to develop ILN metastasis. Prophylactic ILN dissection is recommended in these patients. Prospective studies with larger sample sizes and are needed to support our findings.

\section{Abbreviations}

ILN :inguinal lymph node; LVI: lymphatic vascular infiltration; SD :standard deviation; HBP: high blood pressure; CDV: cardiovascular disease; Cl: confidence interval; HR: hazard ratio;

\section{Declarations}

\section{Acknowledgements}

We thank Jie Liu, PhD ( Department of Vascular and Endovascular Surgery, Chinese PLA General Hospital ) for his helpful review and comments regarding the manuscript.

\section{Availability of data and materials}




\section{Authors' contributions}

Yatao Jia, Yanbo Wang, Yingyi Li conceived and designed the study; Yatao Jia, Yanbo Wang performed the study; Yatao Jia, Hongwei Zhao, Yun Hao,Jiang Zhu analyzed the data; all the authors wrote the article. All authors have read and approved the final manuscript.

\section{Ethics approval and consent to participate}

All procedures performed in studies involving human participants were in accordance with the ethical standards of the institutional research committee and with the 1964 Helsinki Declaration and its later amendments or comparable ethical standards. Informed consent was obtained from all individual participants included in the study.

\section{Competing interests}

The authors have no conflict of interest to declare.

\section{References}

1. Hernandez BY, Barnholtz-Sloan J, German RR, Giuliano A, Goodman MT, King JB, Negoita S, VillalonGomez JM: Burden of invasive squamous cell carcinoma of the penis in the United States, 19982003. Cancer 2008, 113(10 Suppl):2883-2891.

2. Douglawi A, Masterson TA: Updates on the epidemiology and risk factors for penile cancer. Trans/ Androl Urol 2017, 6(5):785-790.

3. Wen S, Ren W, Xue B, Fan Y, Jiang Y, Zeng C, Li Y, Zu X: Prognostic factors in patients with penile cancer after surgical management. World J Urol 2018, 36(3):435-440.

4. Leijte JA, Valdés Olmos RA, Nieweg OE, Horenblas S: Anatomical mapping of lymphatic drainage in penile carcinoma with SPECT-CT: implications for the extent of inguinal lymph node dissection. Eur Urol 2008, 54(4):885-890.

5. Yuan P, Zhao C, Liu Z, Ou Z, He W, Cai Y, Wang Y, Zu X, Qi L, Wang L: Comparative Study of Video Endoscopic Inguinal Lymphadenectomy Through a Hypogastric vs Leg Subcutaneous Approach for Penile Cancer. J Endourol 2018, 32(1):66-72.

6. Zhou X, Zhong Y, Song L, Wang Y, Wang Y, Zhang Q, Cong R, Ji C, Yu T, Song N: Nomograms to predict the presence and extent of inguinal lymph node metastasis in penile cancer patients with clinically positive lymph nodes. Trans/ Androl Urol 2020, 9(2):621-628. 
7. Nascimento A, Pinho JD, Júnior A, Larges JS, Soares FM, Calixto JRR, Coelho RWP, Belfort MRC, Nogueira LR, da Cunha IW et al: Angiolymphatic invasion and absence of koilocytosis predict lymph node metastasis in penile cancer patients and might justify prophylactic lymphadenectomy. Medicine (Baltimore) 2020, 99(9):e19128.

8. Paner GP, Stadler WM, Hansel DE, Montironi R, Lin DW, Amin MB: Updates in the Eighth Edition of the Tumor-Node-Metastasis Staging Classification for Urologic Cancers. Eur Uro/ 2018, 73(4):560-569.

9. Velazquez EF, Ayala G, Liu H, Chaux A, Zanotti M, Torres J, Cho SI, Barreto JE, Soares F, Cubilla AL: Histologic grade and perineural invasion are more important than tumor thickness as predictor of nodal metastasis in penile squamous cell carcinoma invading 5 to $10 \mathrm{~mm}$. Am J Surg Pathol 2008, 32(7):974-979.

10. Pizzocaro G, Algaba F, Horenblas S, Solsona E, Tana S, Van Der Poel H, Watkin NA: EAU penile cancer guidelines 2009. Eur Uro/ 2010, 57(6):1002-1012.

11. Hakenberg OW, Compérat EM, Minhas S, Necchi A, Protzel C, Watkin N: EAU guidelines on penile cancer: 2014 update. Eur Urol 2015, 67(1):142-150.

12. Zhu Y, Ye DW: Lymph node metastases and prognosis in penile cancer. Chin J Cancer Res 2012, 24(2):90-96.

13. Hughes B, Leijte J, Shabbir M, Watkin N, Horenblas S: Non-invasive and minimally invasive staging of regional lymph nodes in penile cancer. World J Urol 2009, 27(2):197-203.

14. Walsh PC, Retik AB, Stamey TA, et al. CamPbell's uorlogy.Vol3.7thed.2453-2478.

15. Hu J, Cui Y, Liu P, Zhou X, Ren W, Chen J, Zu X: Predictors of inguinal lymph node metastasis in penile cancer patients: a meta-analysis of retrospective studies. Cancer Manag Res 2019, 11:64256441.

16. Zhu Y, Zhang SL, Ye DW, Yao XD, Jiang ZX, Zhou XY: Predicting pelvic lymph node metastases in penile cancer patients: a comparison of computed tomography, Cloquet's node, and disease burden of inguinal lymph nodes. Onkologie 2008, 31(1-2):37-41.

17. Tang Y, Rao S, Yang C, Hu Y, Sheng R, Zeng M: Value of MRI morphologic features with pT1-2 rectal cancer in determining lymph node metastasis. J Surg Oncol 2018, 118(3):544-550.

18. Fraley EE, Zhang G, Manivel C, Niehans GA: The role of ilioinguinal lymphadenectomy and significance of histological differentiation in treatment of carcinoma of the penis. J Uro/ 1989, 142(6):1478-1482.

19. Ornellas AA, Nóbrega BL, Wei Kin Chin E, Wisnescky A, da Silva PC, de Santos Schwindt AB: Prognostic factors in invasive squamous cell carcinoma of the penis: analysis of 196 patients treated at the Brazilian National Cancer Institute. J Urol 2008, 180(4):1354-1359.

20. Ornellas AA, Kinchin EW, Nóbrega BL, Wisnescky A, Koifman N, Quirino R: Surgical treatment of invasive squamous cell carcinoma of the penis: Brazilian National Cancer Institute long-term experience. J Surg Onco/ 2008, 97(6):487-495.

21. Solsona E, Iborra I, Rubio J, Casanova JL, Ricós JV, Calabuig C: Prospective validation of the association of local tumor stage and grade as a predictive factor for occult lymph node 
micrometastasis in patients with penile carcinoma and clinically negative inguinal lymph nodes. $J$ Urol 2001, 165(5):1506-1509.

22. Horenblas S, van Tinteren H: Squamous cell carcinoma of the penis. IV. Prognostic factors of survival: analysis of tumor, nodes and metastasis classification system. J Uro/ 1994, 151(5):12391243.

23. Theodorescu D, Russo P, Zhang ZF, Morash C, Fair WR: Outcomes of initial surveillance of invasive squamous cell carcinoma of the penis and negative nodes. J Urol 1996, 155(5):1626-1631.

24. Peak TC, Russell GB, Dutta R, Rothberg MB, Chapple AG, Hemal AK: A National Cancer Databasebased nomogram to predict lymph node metastasis in penile cancer. BJU Int 2019, 123(6):10051010.

25. Diorio GJ, Leone AR, Spiess PE: Management of Penile Cancer. Urology 2016, 96:15-21.

26. Hughes BE, Leijte JA, Kroon BK, Shabbir MA, Swallow TW, Heenan SD, Corbishley CM, van Boven HH, Perry MJ, Watkin NA et al: Lymph node metastasis in intermediate-risk penile squamous cell cancer: a two-centre experience. Eur Urol 2010, 57(4):688-692.

27. Bhagat SK, Gopalakrishnan G, Kekre NS, Chacko NK, Kumar S, Manipadam MT, Samuel P: Factors predicting inguinal node metastasis in squamous cell cancer of penis. World J Uro/ 2010, 28(1):9398.

28. de Paula AA, Netto JC, Freitas R, Jr., de Paula LP, Mota ED, Alencar RC: Penile carcinoma: the role of koilocytosis in groin metastasis and the association with disease specific survival. J Urol 2007, 177(4):1339-1343; discussion 1343.

\section{Figures}




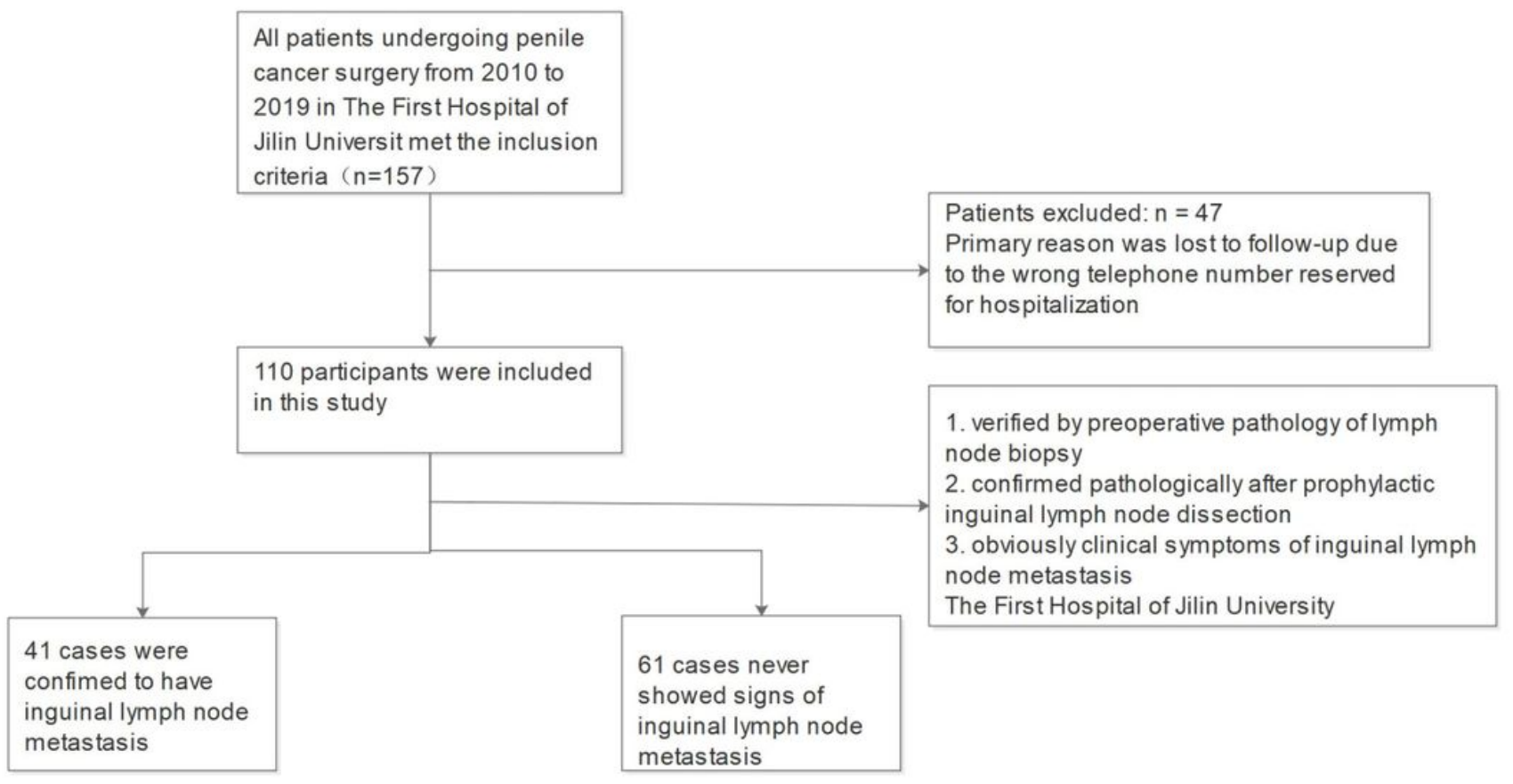

Figure 1

Flow chart 

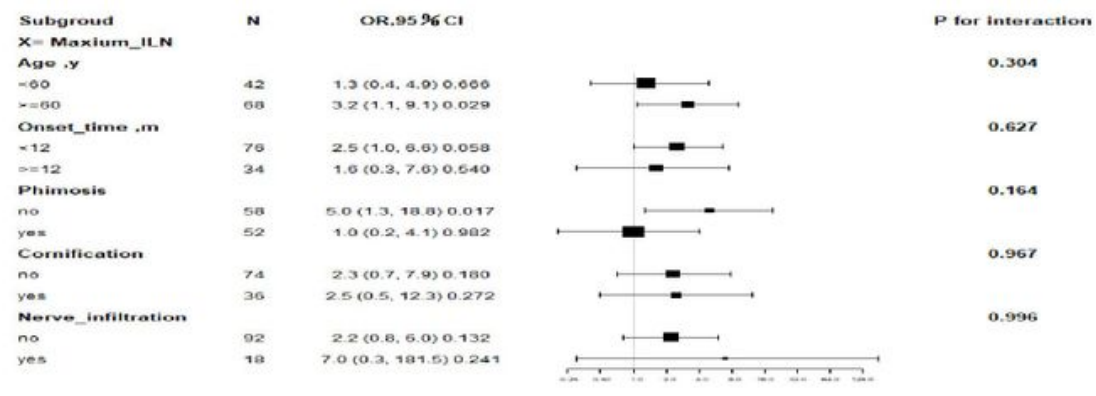

a.
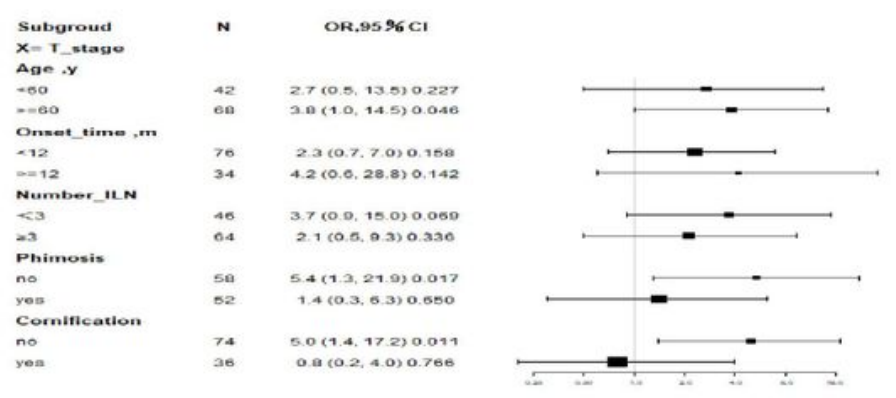

P for interaction

อ.รa

0.971

o.5es

0.446

0.069

b.
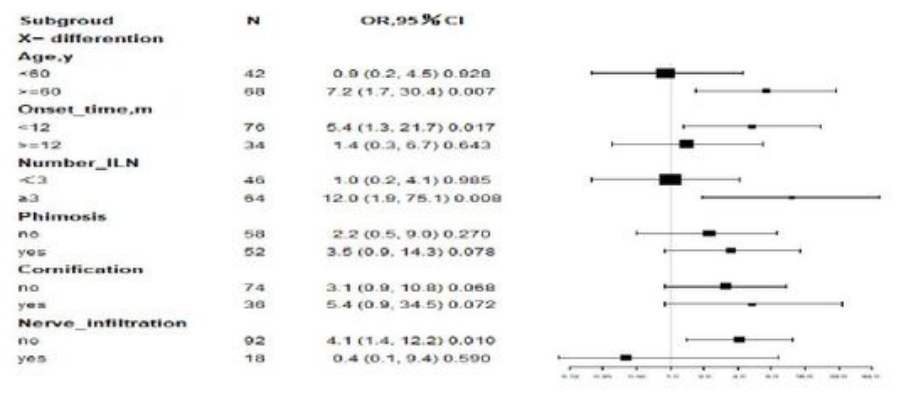

P for interaction

0.12

0.09

0.148

0.62

0.634

0.551

c.
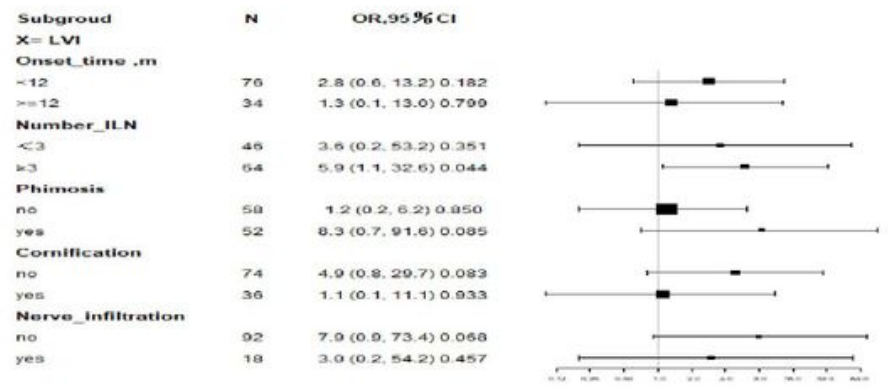

P for interaction

0.631

0.702

0.201

0.111

0.138

d.

\section{Figure 2}

Odds ratios for inguinal lymph node metastasis, according to baseline characteristics.

\section{Supplementary Files}

This is a list of supplementary files associated with this preprint. Click to download. 
- Additionalfile1.xlsx

Page 16/16 